\title{
2011 appropriate use criteria audit of an echocardiography lab in South Western Nigeria
}

\author{
Opeyemi O. Oni, ${ }^{1}$ Moshood A. Adeoye, ${ }^{2}$ Adewole Adebiyi, ${ }^{2}$ Akinyemi Aje, ${ }^{2}$ Olaniyi Oyebowale, ${ }^{3}$ \\ Saheed O. Adebayo, ${ }^{4}$ Julius O. Adesina, ${ }^{5}$ Okechukwu O. Ogah, ${ }^{2}$ Eniola A. Bamgboye, ${ }^{6}$ \\ M. David Dairo, ${ }^{6}$ Iseko I. Iseko, ${ }^{7}$ Godwin J. Emeka ${ }^{8}$
}

${ }^{1}$ Department of Medicine, Bowen University Teaching Hospital, Ogbomoso; ${ }^{2}$ Division of Cardiovascular Medicine, Department of Medicine, University College Hospital, Ibadan; ${ }^{3}$ Division of Cardiology, Department of Medicine, State Hospital, Abeokuta; ${ }^{4}$ Division of Cardiology, Department of Internal Medicine, Federal Medical Centre, Abeokuta; ${ }^{5}$ Cardiology Unit, Department of Internal Medicine, Federal Medical centre, Abeokuta; ${ }^{6}$ Department of Epidemiology and Medical Statistics, Faculty of Public health, University of Ibadan; ${ }^{7}$ CardioCare Specialty Hospital, Limi Hospital Group, Abuja; ${ }^{8}$ Department of Chemical pathology, University of Ibadan, Ibadan, Nigeria

\begin{abstract}
Cardiovascular diseases are the major cause of death worldwide. Since its discovery in the 20th century, Echocardiography (ECHO) has become one of the pivotal tools in assessing cardiac
\end{abstract}

Correspondence: Opeyemi O. Oni, Department of Medicine, Bowen University Teaching Hospital, P.M.B. 4002 and P.O.Box, 15, Ogbomoso, Nigeria.

Tel.: +234(0)8058435811

E-mail: oniopeyemi64@gmail.com

Key words: Appropriate use criteria; hypertension; hypertensive heart disease; heart failure; left ventricular geometry.

Acknowledgment: Technical support offered by the staff of the Echocardiography Suite deeply appreciated.

Conflict of interest: The authors have no conflict of interest to declare.

Availability of data and materials: All data generated or analyzed during this study are included in this published article.

Ethics approval and consent to participate: The Ethics Committee of $\mathrm{UI} / \mathrm{UCH}$ Institutional Review Board approved this study (UI/EC/16/0168). The study is conformed with the Helsinki Declaration of 1964, as revised in 2013, concerning human and animal rights. All patients participating in this study signed a written informed consent form for participating in this study.

Informed consent: Written informed consent was obtained from a legally authorized representative(s) for anonymized patient information to be published in this article.

Received for publication: 7 December 2020.

Revision received: 14 May 2020.

Accepted for publication: 14 May 2020.

This work is licensed under a Creative Commons Attribution NonCommercial 4.0 License (CC BY-NC 4.0).

${ }^{(C)}$ Copyright: the Author(s), 2021

Licensee PAGEPress, Italy

Annals of Clinical and Biomedical Research 2021; 2:128

doi:10.4081/acbr.2021.128 structure and function. With the increase in requests for $\mathrm{ECHO}$, there has risen an unwanted problem - inappropriate requests for ECHO. There has therefore arisen the need to audit ECHO labs for the appropriateness of ECHO requests. The patients referred from the outpatient clinics and in-patient wards for ECHO from June $1^{\text {st, }}$ 2015 till September $30^{\text {th }}, 2016$ were recruited. Their request form data, clinical information, and ECHO results were analyzed as appropriate. The 2011 appropriate use criteria for Transthoracic ECHO was utilized. The most common indication out of the 2174 ECHOs reviewed was hypertension (16\%), closely followed by hypertensive heart disease $(12.4 \%)$. The percentage of appropriate, inappropriate, and uncertain indications according to the 2011 appropriate use criteria (AUC) for transthoracic echocardiography were $41.4 \%, 31.1 \%$, and $0.1 \%$ respectively. Less than ten percent $(9.3 \%)$ of the indications could not be classified by the 2011 AUC while $18.1 \%$ of the ECHOs had no indication. When indications of Hypertension, Hypertensive Heart Disease (HHD) and heart failure were compared, heart failure was significantly associated with eccentric Left Ventricular Hypertrophy (LVH), larger LV mass, lower BMI, larger cardiac dimensions, reduced ejection fraction, lower trans mitral A velocities than the other two indications. Concentric LVH was showed a trend towards being most in those with HHD $(p=0.072)$. The percentage of appropriate indications was low in this study as compared to others, largely because of large inappropriate indications. There is a need to ensure appropriate indications are filled for ECHO request forms. The 2011 AUC may need to be reviewed to expand the appropriate group of indications.

\section{Introduction}

Transthoracic Echocardiography (ECHO) has evolved since its discovery in the 1950 s as a simple, non-invasive, relatively cheap and objective tool for assessing cardiac function. ${ }^{1}$ Transoesophageal echocardiography also evolved a few decades ago, which has increased spatial resolution with an improved evaluation of the atrial appendages and the mitral valvular apparatus.

The relatively low cost, non-invasiveness, minimal patient discomfort involved and the real-time imaging of cardiac structures and function of echocardiography makes it an invaluable tool in cardiovascular imaging as compared to other modalities like ventriculography, cardiac computed tomography imaging and magnetic resonance imaging. Apart from cardiac specific conditions, 
diverse systemic problems could also benefit from Echo, including stroke, Ehler-Danlos syndrome, Marfan's syndrome, adult polycystic kidney disease among others. ${ }^{2-5}$

The presence of various risk factors among people groups and the recognition of various indices of cardiac dysfunction prompts referrals for echocardiography. Also, the anticipation of a possible change in cardiovascular status following an intervention like the administration of cytotoxic drugs may prompt referrals for ECHO. The preoperative cardiovascular assessment of patients may also warrant the referral for echo as required.

Nigeria is blessed with a population of about one hundred and seventy-five million people and has an array of cardiovascular risk factors for which people seek proper cardiovascular evaluation. ${ }^{6}$ History taking and physical examination have been used as invaluable screening tools over the years for various risk factors. However, history taking and physical examination are limited and there is a need for improvement in cardiovascular assessment and diagnosis of on-going or established target organ damage so as to institute appropriate levels of prevention and intervention.

It is also noteworthy, however, that a tool with such wide applicability like ECHO is prone to abuse and misuse. It has been speculated that where ready aids like ECHO and electrocardiography exist, the mind goes to sleep. Thus, what should have been a complement to sound reasoning following proper history and physical exam now becomes the primary screening tool. This breeds what has been appropriately termed hyposkillia-deficiency of clinical skills which is covered up by use of investigations. ${ }^{7}$ This contributes on the long run to overburdening of the health sector, incurring of undue cost for both patients and cardiologists, and brain drain.

The American college of cardiology foundation developed an appropriate use criteria for evaluating requests for Transthoracic (TTE) and Transoesophageal Echocardiography (TEE), first in 2007 and now 2011. ${ }^{8}$ This has been used in North America and Europe but not yet in West Africa to the best of our knowledge. We therefore set out to use the same tool to evaluate our practice in Nigeria, Africa's most populous nation.

\section{Materials and Methods}

The study was carried out at the Cardiology Unit of the University College Hospital (UCH). University College Hospital is located in Ibadan, the capital city of Oyo state. It is the Premier Teaching Hospital in Nigeria and the major tertiary centre in the state, sub-serving the whole state and neighbouring states within the South West geopolitical zone. Most clinical specialties refer patients for echocardiography for various reasons.

Echocardiography commenced in $\mathrm{UCH}$ about 2 decades ago and has become a cornerstone tool in evaluating cardiac structure and function. The ECHO suite is presently well-staffed with physiologists, cardiac nurses and cardiologists. Echocardiography is done routinely every Wednesday, though it is also done on any day of the week for emergencies, as occasion demands.

Eligible participants in the study were people referred for echocardiography to the ECHO suite of UCH, Ibadan. The study was a descriptive, cross-sectional study involving a review of the records of all requests and services provided by the unit over a period of sixteen months. Those included were individuals aged 12 years and above who were referred for echocardiography to the ECHO suite of UCH, Ibadan. Those aged less than 12 years at the time of echocardiographic evaluation and patients with incomplete data were excluded from the study.
A total sampling method was used, which essentially consisted of all the patients that were referred for ECHO from June 2015 till September 2016. All those who satisfied the inclusion criteria were incorporated into the study. The ethical approval was obtained from the joint University of Ibadan and University College Hospital ethical review board. This research conformed to widely accepted ethical principles as stated at the declaration of Helsinki.

The cardiac structure and function of the subjects and controls were evaluated at the ECHO Suite of the hospital. Data were collected using a proforma consisting of the following sections: Demographics, anthropometric information, indications for referral, cadre of doctor referring patient and Echocardiographic variables, including the diagnosis. Measurements were made according to the recommendations of the American Society of Echocardiography. ${ }^{9}$

\section{Data management}

Data were collected using a standard proforma. Analysis was done using the Statistical Package for Social Sciences (SPSS) version 20. Continuous variables were expressed as means and categorical variables expressed as percentages. Differences in categorical variables were assessed using the chi-square test while that of continuous variables was evaluated using T-test and Analysis of Variance (ANOVA) test. A two-tailed $\mathrm{P}$ value $<0.05$ was considered significant.

\section{Results}

A total of 2174 echocardiography studies were done. Out of these, 394 people were referred for Echocardiography with no indications for the test on their request cards. The appropriate use criteria - 2011 edition- has a total of 98 indications for transthoracic echocardiography. These are categorized into appropriate, inappropriate and uncertain. A total of 34 indications were applicable to this ECHO lab. These are listed below in Table 1.

Seven hundred and thirty-three $(33.7 \%)$ of echocardiography tests done were not classified as $339(15.6 \%)$ of these had indications that were not addressed by the 2011 AUC document. The other 394 (18.1\%) requests had no indication for echocardiography on the request forms. Of the 1578 ECHOs that could be evaluated by the 2011 appropriate use criteria, $56.9 \%$ of them were appropriate, $43 \%$ were inappropriate and 0.001 were uncertain. Table 2 shows the ECHOs addressed by the 2011 AUC, including those with no indications and those that could not be classified by the AUC.

Table 3 shows the characteristics of the ECHO results according to the indications for ECHO. Those with appropriate, inappropriate and unclassified indications had $23.6 \%, 24 \%$ and $27.9 \%$ normal results respectively. Those with appropriate indications clearly showed a significant difference between those with normal and those with abnormal ECHOs in terms of their systolic blood pressures, left ventricular ejection fractions and their left atrial sizes. Other details can be seen in Table 3 .

Cardiologists were the chief source of echocardiogram referrals, followed by surgeons. About a third of the referrals could not be traced to any particular source due to incompleteness of records.

Those in the inappropriate category were generally older, had higher Body Mass Index (BMI), higher blood pressure and pulse pressure values than the other categories. Subjects in the appropriate category had larger left atrial diameters, lower ejection fractions, larger left ventricular volumes and heavier left ventricles than others. Details can be seen in Table 4. 


\section{Hypertension, hypertensive heart disease and heart failure}

Those with hypertension were younger than those with Hypertensive Heart Disease (HHD), with a mean age difference of 4 years. Twenty percent $(20 \%)$ of those with hypertension had left atrial enlargement, as compared to HHD (37.1\%) and heart failure $(65 \% ; \mathrm{p}=0.000)$. Those with hypertension weighed more, had higher body surface area, lower left ventricular mass and higher left ventricular ejection fraction as compared to those with hypertensive heart disease. Details can be seen in Table 5 .

Those with heart failure had lower systolic and diastolic blood pressures, larger cardiac dimensions, higher LV mass, lower ejection fraction, slower trans-mitral A velocities and shorter E deceleration time. Details can be seen in Table 6 .

\section{Geometric patterns among hypertension, hypertensive heart disease and heart failure}

Those with heart failure had significantly less normal geomet- ric patterns as compared to either hypertension or hypertensive heart disease, especially in women $(\mathrm{p}=0.000)$. Concentric remodelling was however relatively common, especially among men with hypertension $(\mathrm{P}=0.000)$. Eccentric LVH was significantly more in those with heart failure among either gender $(\mathrm{p}=0.000$ for men and women). Concentric LVH was not different whithin the three classes, though showing a trend towards being highest in those with hypertensive heart disease $(\mathrm{p}=0.072)$.

\section{Discussion}

A total of 2174 ECHO studies were recruited in this study. Hypertension was the most common indication for ECHO (16\%). This is comparable to the findings of Oyedeji et al., where hypertension was the most common indication for ECHO. ${ }^{10}$ The percentage of $16.1 \%$ is however much lower than that in Cuspidi et al. $(30.4 \%) .{ }^{11}$ This is likely due to the fact that Hypertension and

Table 1. Indications for echocardiography at the ECHO suite, University College Hospital.

\begin{tabular}{|c|c|c|}
\hline Number of indication & Summary of indication & N (\%) \\
\hline 1 & Symptoms or conditions potentially related to suspected cardiac aetiology & $240(11)$ \\
\hline 2 & Prior testing that is concerning for heart disease or structural abnormality & $120(5.5)$ \\
\hline 4 & Frequent VPCs or exercise induced VPCs & $1(0)$ \\
\hline 5 & Sustained or non-sustained atrial fibrillation, SVT or VT & $8(0.4)$ \\
\hline 6 & Asymptomatic isolated sinus bradycardia & $17(0.8)$ \\
\hline 7 & Clinical symptoms or signs consistent with a cardiac diagnosis known to cause light headedness/pre-syncope/syncope & $5(0.2)$ \\
\hline 9 & Syncope with no symptoms/signs of CV disease & $3(0.1)$ \\
\hline 13 & Routine perioperative evaluation of ventricular function with no symptoms/signs of CV disease & $227(10.4)$ \\
\hline 15 & Evaluation of suspected pulmonary hypertension, RV function and Pulmonary artery pressure & $12(0.6)$ \\
\hline 21 & Acute chest pain with suspected MI and non-diagnostic ECG & $2(0.1)$ \\
\hline 28 & Suspected Pulmonary embolism in order to establish diagnosis & $2(0.1)$ \\
\hline 29 & Known acute pulmonary embolism to guide therapy & $7(0.3)$ \\
\hline 31 & Re-evaluation of known pulmonary embolism after thrombolysis or thrombectomy & $1(0.0)$ \\
\hline 34 & Initial evaluation when there is a reasonable suspicion of valvular or structural heart disease & $5(0.2)$ \\
\hline 37 & Re-evaluation of known valvular heart disease with a change in clinical status to guide therapy & $1(0.0)$ \\
\hline 47 & Initial post-op evaluation of prosthetic valve & $1(0.0)$ \\
\hline 52 & Initial evaluation of suspected infective endocarditis with positive blood cultures/a new murmur & $3(0.1)$ \\
\hline 55 & Re-evaluation of infective endocarditis at high risk of progression/a change in clinical status & $1(0.0)$ \\
\hline 57 & Suspected Cardiac mass & $2(0.1)$ \\
\hline 58 & Suspected cardiovascular source of embolus & $1(0.0)$ \\
\hline 59 & Suspected pericardial conditions & $2(0.1)$ \\
\hline 61 & Re-evaluation of known pericardial effusion to guide management or therapy & $6(0.3)$ \\
\hline 63 & Evaluation of the ascending aorta in the setting of a known or suspected connective tissue disease & $2(0.1)$ \\
\hline 67 & Initial evaluation of suspected hypertensive heart disease & $186(8.6)$ \\
\hline 68 & Routine evaluation of systemic hypertension without symptoms or signs of hypertensive heart disease & $351(16)$ \\
\hline 70 & Initial evaluation of known or suspected HF (systolic and diastolic) based on symptoms or signs & 124(5.7) \\
\hline 71 & Re-evaluation of known HF with a change in clinical status & $1(0.0)$ \\
\hline 72 & Re-evaluation of the known HF with a clear precipitating change in medication or diet & $2(0.1)$ \\
\hline 73 & Re-evaluation of known HF to guide therapy & $2(0.1)$ \\
\hline 79 & Routine surveillance of implanted device without a change in clinical status or cardiac exam & $1(0.0)$ \\
\hline 86 & Initial evaluation of known or suspected cardiomyopathy & $21(1)$ \\
\hline 87 & Re-evaluation of known cardiomyopathy with change in clinical status or cardiac exam or to guide therapy & $2(0.1)$ \\
\hline 91 & Baseline and serial re-evaluations in a patient undergoing therapy with cardio toxic agents & $70(3.2)$ \\
\hline 92 & Initial evaluation of known or suspected adult congenital heart disease & $3(0.1)$ \\
\hline
\end{tabular}


hypertensive heart disease are separate indications in this study (as compared to theirs), with both having a combined prevalence of $24.6 \%$. Oyedeji et al. considered hypertension and hypertensionrelated indications together and reported a joint prevalence of $38.1 \%$ in their study. They worked in a similar geopolitical zone (just $133 \mathrm{~km}$ south of our centre). However, their higher prevalence may be due to the type of clinical practice - theirs is a private clinic with a total number of 168 cases over a 2 -years period while ours is a public, tertiary hospital which grossed over $1200 \%$ of their number of patients, cutting across most disciplines of medicine. The sizable number of referrals for surgeons, family physicians and other disciplines may have accounted for a salutary effect on the prevalence of hypertension and hypertension-related indications in this study.

The next most common indication is that of symptoms suspect of a potential cardiac aetiology $(11 \%)$. These consisted of stroke, palpitations, breathlessness, pedal swelling, among others. Other common indications include preoperative cardiac evaluation $(10.4 \%)$, evaluation of cardiac function in those about to commence cardiotoxic agents $(5.7 \%)$, prior testing that is concerning heart disease $(5.5 \%)$ and heart failure (3.2\%).
The appropriateness of the indications for echocardiography at the $\mathrm{ECHO}$ suite

Most studies have shown that most Transthoracic echocardiogram labs largely comply with 2011 AUC criteria, with greater than $80 \%$ of the indications being appropriate. ${ }^{12-14}$ However, this study found that only $41.1 \%$ of the indications for ECHO were appropriate. This is in stark contrast to the findings from most studies. This could be possibly explained by the unduly high proportion of those ECHO requests that had no indication. If these were taken out, the adjusted prevalence of appropriate indications will be $50.4 \%$. Another reason is that the most common indication for ECHO in our lab, hypertension, is classified as inappropriate

Table 2. Appropriateness of indications for Echocardiography.

\begin{tabular}{lc} 
Indication & $\mathbf{N}(\%)$ \\
Appropriate & $898(41.3)$ \\
Inappropriate & $678(31.1)$ \\
\hline Unclassified & $203(9.3)$ \\
No indication & $394(18.1)$ \\
\hline Uncertain & $2(0.1)$ \\
\hline
\end{tabular}

Table 3. Classification of ejection fraction, left atrial diameter and blood pressure according to the appropriateness of the indication for echocardiography.

\begin{tabular}{|c|c|c|c|}
\hline Appropriate-Variables & Normal study $(n=210)$ & Abnormal study ( $\mathrm{n}=681$ ) & P value \\
\hline Ejection fraction (\%) & $68.7 \pm 8.6$ & $58.9 \pm 18.2$ & 0.000 \\
\hline Left atrial diameter $(\mathrm{cm})$ & $3.31 \pm 0.46$ & $3.86 \pm 0.81$ & 0.000 \\
\hline Systolic blood pressure (mmHg) & $127.8 \pm 18.8$ & $132.4 \pm 22.5$ & 0.025 \\
\hline Inappropriate-Variables & Normal study $(\mathrm{n}=115)$ & Abnormal study $(\mathrm{n}=364)$ & P value \\
\hline Ejection fraction (\%) & $68.9 \pm 9.54$ & $68.4 \pm 12.8$ & 0.61 \\
\hline Left atrial diameter $(\mathrm{cm})$ & $3.37 \pm 0.42$ & $3.59 \pm 0.58$ & 0.000 \\
\hline Systolic blood pressure (mmHg) & $136.4 \pm 18.6$ & $142.1 \pm 21.6$ & 0.011 \\
\hline Unclassified-Variables & Normal study ( $\mathrm{n}=39$ ) & Abnormal study ( $\mathrm{n}=101$ ) & P value \\
\hline Ejection fraction (\%) & $70.3 \pm 7.1$ & $66.3 \pm 12.9$ & 0.035 \\
\hline Left atrial diameter $(\mathrm{cm})$ & $2.77 \pm 0.39$ & $2.87 \pm 0.4$ & 0.107 \\
\hline Systolic blood pressure (mmHg) & $127.3 \pm 16.6$ & $130.2 \pm 20.4$ & 0.421 \\
\hline
\end{tabular}

Table 4. Anthropometric, clinical and echocardiographic variables among the major appropriate use criteria classes.

\begin{tabular}{|c|c|c|c|c|c|}
\hline Variables & Appropriate $n=898$ & Inappropriate $\mathrm{n}=678$ & Unclassified $n=203$ & F static & P value \\
\hline Age (years) & $54.0 \pm 17.1$ & $58.6 \pm 14.0$ & $52.6 \pm 18.1$ & 19.407 & 0.000 \\
\hline Systolic BP (mmHg) & $131.2 \pm 21.8$ & $140.8 \pm 20.9$ & $129.2 \pm 19.6$ & 32.639 & 0.000 \\
\hline Diastolic BP (mmHg) & $83.0 \pm 13.3$ & $86.9 \pm 13.5$ & $81.6 \pm 12.8$ & 15.089 & 0.000 \\
\hline Aortic root Diameter $(\mathrm{cm})$ & $2.85 \pm 0.43$ & $2.87 \pm 0.42$ & $2.84 \pm 0.40$ & 0.741 & 0.477 \\
\hline Left atrial diameter $(\mathrm{cm})$ & $3.72 \pm 0.78$ & $3.54 \pm 0.55$ & $3.54 \pm 0.71$ & 16.999 & 0.000 \\
\hline Stroke volume $\left(\mathrm{cm}^{3}\right)$ & $70.8 \pm 25.5$ & $73.1 \pm 24.6$ & $67.3 \pm 24.7$ & 4.479 & 0.011 \\
\hline Fractional shortening (\%) & $34.6 \pm 12.0$ & $39.4 \pm 9.4$ & $38.2 \pm 9.7$ & 39.592 & 0.000 \\
\hline Ejection Fraction (\%) & $61.3 \pm 17.1$ & $68.5 \pm 12.2$ & $67 \pm 12.7$ & 47.731 & 0.000 \\
\hline Body mass index $\left(\mathrm{Kg} / \mathrm{m}^{2}\right)$ & $27.0 \pm 6.2$ & $27.8 \pm 5.9$ & $26.5 \pm 5.4$ & 4.796 & 0.008 \\
\hline Pulse Pressure (mmHg) & $48.2 \pm 16.42$ & $53.8 \pm 16.4$ & $47.6 \pm 14.5$ & 18.529 & 0.000 \\
\hline Height (m) & $1.65 \pm 0.1$ & $1.65 \pm 0.1$ & $1.64 \pm 0.1$ & 3.957 & 0.019 \\
\hline LV mass indexed to Height ${ }^{2.7}\left(\mathrm{~g} / \mathrm{m}^{2.7}\right)$ & $45.1 \pm 19.7$ & $42.8 \pm 15.2$ & $41 \pm 17.4$ & 5.341 & 0.005 \\
\hline $\mathrm{LV}$ mass indexed to $\mathrm{BSA}\left(\mathrm{g} / \mathrm{m}^{2}\right)$ & $97.3 \pm 42.3$ & $90.2 \pm 30.4$ & $87.0 \pm 34.9$ & 9.129 & 0.000 \\
\hline BSA (Dubois) $\left(\mathrm{m}^{2}\right)$ & $1.81 \pm 0.2$ & $1.82 \pm 0.2$ & $1.76 \pm 0.2$ & 5.198 & 0.006 \\
\hline LV Volume BSA $\left(\mathrm{cm}^{3}\right)$ & $70.1 \pm 33.4$ & $60.9 \pm 21.5$ & $59.4 \pm 25.9$ & 22.145 & 0.000 \\
\hline
\end{tabular}


by the AUC 2011. This causes a sharp fall in the percentage of appropriate indications.

Two hundred and three ECHOs were done which could not be classified by the 2011 AUC. Some of these indications include cancer, chronic kidney disease, prostate enlargement, simple multi nodular goitre, sickle cell anaemia, suspected COPD, Ischaemic heart disease, routine $\mathrm{ECHO}$, suspected stroke and diabetes mellitus, among others. While it is true that some of these conditions may get complicated and require $\mathrm{ECHO}$, they do not qualify as standalone indications, nor were they addressed as being either inappropriate or uncertain in the 2011 AUC.

An unpleasant finding is that of 394 ECHOs being done at the lab with absolutely no indication. This is more in number than the highest-ranking indication for ECHO (16\% vs18.1\%) This has not been commonly reported.

\section{The correlation between the indications and the results of the ECHO}

All classifications of indications for ECHO revealed normal findings, with appropriate, inappropriate and unclassified yielding percentages of $23.6 \%, 24 \%$, and $27.9 \%$ respectively. The results of the ECHOs done for those with appropriate indications showed a significant difference in the systolic blood pressure, left ventricular ejection fraction and left atrial diameter among those with normal as compared to those with abnormal findings. This suggests that those with abnormal findings on ECHO had significantly higher blood pressure readings than their counterparts, had reduced systolic function and had larger left atrial diameters - a measure of diastolic dysfunction. ${ }^{15}$ It is interesting to note that this pattern is not found in the other classifications, as those with inappropriate indications had similar left ventricular ejection fractions regardless of whether the ECHO report ended up being normal or not. However, for those who had abnormal ECHO results, the systolic blood pressure was significantly higher as compared to those with normal results. This suggests that there may be a yet undefined blood pressure cut-off point at which ECHO may be indicatedregardless of the duration of hypertension or presence of other risk factors. This also may be a fall out of the fact that Hypertension, the most common indication for ECHO in this lab, was classified as inappropriate according to the 2011 AUC guideline.

Those with an unclassified indication for ECHOs had significantly lower left ventricular ejection fractions among those with abnormal ECHO findings as compared with those who had normal ECHOs. However, their left atrial diameters and systolic blood pressures were essentially the same in both groups.

The summary of this is that the appropriate indications have good discriminant ability, with those having abnormal ECHO findings having elevated blood pressure as compared to those with normal findings. The findings from inappropriate indications' analysis suggest that there may be a yet to be defined cut off point, at which

Table 5. Comparison between those with indications of hypertension and hypertensive heart disease.

\begin{tabular}{|c|c|c|c|}
\hline Variables & e heart disease $n=170$ & Hypertension $n=325$ & P value \\
\hline Height (cm) & $164.9 \pm 9.7$ & $165.2 \pm 9.5$ & 0.673 \\
\hline Weight (kg) & $74.5 \pm 14.1$ & $78.1 \pm 15.9$ & 0.013 \\
\hline Age (years) & $60.4 \pm 14.1$ & $56.4 \pm 13.5$ & 0.001 \\
\hline Systolic BP (mmHg) & $140.3 \pm 22.7$ & $143.3 \pm 20.4$ & 0.183 \\
\hline Diastolic BP (mmHg) & $86.1 \pm 14.3$ & $89.1 \pm 13.3$ & 0.036 \\
\hline Aortic root diameter $(\mathrm{cm})$ & $2.9 \pm 0.42$ & $2.87 \pm 0.4$ & 0.318 \\
\hline Aortic valve opening (cm) & $1.97 \pm 0.36$ & $1.97 \pm 0.34$ & 0.890 \\
\hline Left atrial diameter $(\mathrm{cm})$ & $3.8 \pm 0.73$ & $3.6 \pm 0.58$ & 0.000 \\
\hline Left ventricular internal diameter (diastole) $(\mathrm{cm})$ & $4.95 \pm 0.9$ & $4.78 \pm 0.7$ & 0.019 \\
\hline Left ventricular internal diameter (systole) (cm) & $3.3 \pm 1.1$ & $2.9 \pm 0.8$ & 0.000 \\
\hline End diastolic volume $\left(\mathrm{cm}^{3}\right)$ & $121.3 \pm 55.5$ & $109.9 \pm 38.4$ & 0.006 \\
\hline End systolic volume $\left(\mathrm{cm}^{3}\right)$ & $51.6 \pm 48.1$ & $37.8 \pm 27.0$ & 0.000 \\
\hline Stroke volume $\left(\mathrm{cm}^{3}\right)$ & $71.4 \pm 25.0$ & $73.0 \pm 23.7$ & 0.469 \\
\hline Fractional shortening (\%) & $35.5 \pm 11.5$ & $39.1 \pm 9.9$ & 0.000 \\
\hline Ejection fraction (\%) & $63.1 \pm 16.5$ & $67.9 \pm 12.9$ & 0.000 \\
\hline E velocity (m/sec) & $0.75 \pm 0.25$ & $0.70 \pm 0.21$ & 0.023 \\
\hline A velocity (m/sec) & $0.70 \pm 0.23$ & $0.72 \pm 0.20$ & 0.137 \\
\hline E deceleration time (msec) & $188.8 \pm 72.0$ & $178.7 \pm 68.2$ & 0.117 \\
\hline BMI $\left(\mathrm{kg} / \mathrm{m}^{2}\right)$ & $27.7 \pm 6.4$ & $28.7 \pm 6.0$ & 0.076 \\
\hline LV mass (kg) & $183.2 \pm 88.6$ & $166.9 \pm 57.8$ & 0.011 \\
\hline Relative wall thickness & $0.41 \pm 0.13$ & $0.41 \pm 0.12$ & 0.692 \\
\hline End-diastolic volume ${ }^{B S A}\left(\mathrm{~cm}^{3} / \mathrm{m}^{2}\right)$ & $69.4 \pm 31.4$ & $60.6 \pm 20.6$ & 0.000 \\
\hline Pulse pressure (mmHg) & $54.3 \pm 18.9$ & $54.1 \pm 16.1$ & 0.944 \\
\hline Left ventricular mass indexed to height ${ }^{2.7}\left(\mathrm{~g} / \mathrm{m}^{2.7}\right)$ & $48.3 \pm 23$ & $43.3 \pm 14.4$ & 0.004 \\
\hline Body surface area Monstellar (m²) & $1.84 \pm 0.18$ & $1.88 \pm 0.2$ & 0.015 \\
\hline Body surface area Dubois $\left(\mathrm{m}^{2}\right)$ & $1.81 \pm 0.17$ & $1.85 \pm 0.20$ & 0.022 \\
\hline Left ventricular mass ${ }^{\text {BSA }}\left(\mathrm{g} / \mathrm{m}^{2}\right)$ & $102.5 \pm 49.4$ & $90.4 \pm 29.3$ & 0.001 \\
\hline
\end{tabular}


uncontrolled blood pressure may be a stand-alone indication for ECHO. The unclassified indications may be sorted out individually and standardized based on individual merit or demerit.

Over 2000 ECHOs were done during the period of review. Only $41.1 \%$ of the indications were appropriate according to the 2011 AUC, a percentage that is quite low as compared to figures from most parts of the world. ${ }^{16,17}$ The main reason is that hypertension, the most common indication for ECHO in this study, is an inappropriate indication, a point which was further emphasized by recommendations of use of TTE in hypertension. ${ }^{18}$ However, $74 \%$ of people referred with inappropriate indications had left atrial enlargement. Their blood pressures were also significantly higher than those with normal findings on ECHO. Over $18 \%$ of the ECHOs done had no indication whatsoever. Cardiologists are also the chief source of referral for ECHOs to the lab, though accounting for only $27.2 \%$ of all referrals. There is, therefore, a need to educate the healthcare community on what indications are appropriate, while also re-evaluating the 2011 appropriate use criteria itself for possible modifications to properly classify patients. This has been proposed by Fonseca et al. ${ }^{19}$

\section{Hypertension, hypertensive heart disease and heart failure}

Long standing, uncontrolled hypertension progresses inexorable to heart failure and as a result, about $75 \%$ of heart failure patients have a history of hypertension..$^{20,21}$ Hypertensive heart disease is common in Nigeria and hypertension is the most common cause of heart failure-consistently responsible more than $50 \%$ of cases nationwide. ${ }^{22,23}$ Those with Hypertension in this study weighed more, were younger and generally had higher blood pressures than those with heart failure (possibly due to other aetiologies causing heart failure or due to decapitated hypertension). The mean age of patients with heart failure in this study $(58.1 \pm 15.7$ years) is essentially same with those of the Inter-CHF study $\left(59 \pm 0.2\right.$ years). ${ }^{24}$ Those with heart failure had lower BMI (possibly from imminent cardiac cachexia), larger cardiac dimensions, reduced left ventricular ejection fraction, higher trans-mitral $\mathrm{E}$ velocities, lower trans-mitral A velocities and shorter E deceleration rates than those with hypertension and hypertensive heart disease. The trans-mitral E/A ratio and $\mathrm{E}$ deceleration time suggests a pseudo normal filling pattern for those with heart failure. They also had higher left ventricular mass and narrower pulse pressures than the other two groups.

\section{Left ventricular geometry}

Eccentric LVH was the predominant geometry in heart failure cases for either gender (almost $50 \%$ of all heart failure cases) while concentric remodelling was infrequent in heart failure patients, especially in men. Interestingly, Concentric LVH was not significantly more common among the three classes though the absolute prevalence was highest in those with hypertensive heart disease.

The normal LV geometry was unsurprisingly most prevalent in those with hypertension and least prevalent in those with heart failure.

Table 6. Comparison between those with indications of hypertensive heart disease and heart failure.

\begin{tabular}{|c|c|c|c|}
\hline Variables & Hypertensive heart disease $n=170$ & Heart failure $n=114$ & P value \\
\hline Height (cm) & $164.9 \pm 9.7$ & $166.1 \pm 8.9$ & 0.299 \\
\hline Weight (kg) & $74.5 \pm 14.1$ & $74.1 \pm 17.8$ & 0.852 \\
\hline Age (years) & $60.4 \pm 14.1$ & $58.1 \pm 15.7$ & 0.180 \\
\hline Systolic BP (mmHg) & $140.3 \pm 22.7$ & $121.2 \pm 21.3$ & 0.000 \\
\hline Diastolic BP (mmHg) & $86.1 \pm 14.3$ & $79.5 \pm 13.6$ & 0.001 \\
\hline Aortic Root Diameter (cm) & $2.9 \pm 0.42$ & $2.90 \pm 0.45$ & 0.927 \\
\hline Aortic valve opening (cm) & $1.97 \pm 0.36$ & $1.91 \pm 0.31$ & 0.110 \\
\hline Left atrial diameter $(\mathrm{cm})$ & $3.8 \pm 0.73$ & $4.3 \pm 0.88$ & 0.000 \\
\hline Left ventricular internal diameter in diastole $(\mathrm{cm})$ & $4.95 \pm 0.9$ & $5.74 \pm 1.2$ & 0.000 \\
\hline Left ventricular internal diameter in systole (cm) & $3.3 \pm 1.1$ & $4.4 \pm 1.5$ & 0.000 \\
\hline End diastolic volume $\left(\mathrm{cm}^{3}\right)$ & $121.3 \pm 55.5$ & $173.2 \pm 78.1$ & 0.000 \\
\hline End systolic volume $\left(\mathrm{cm}^{3}\right)$ & $51.6 \pm 48.1$ & $103.7 \pm 67.4$ & 0.000 \\
\hline Stroke volume $\left(\mathrm{cm}^{3}\right)$ & $71.4 \pm 25.0$ & $69.7 \pm 27.3$ & 0.572 \\
\hline Fractional shortening (\%) & $35.5 \pm 11.5$ & $25.5 \pm 13.9$ & 0.000 \\
\hline Ejection fraction (\%) & $63.1 \pm 16.5$ & $45.6 \pm 19.5$ & 0.000 \\
\hline E velocity $(\mathrm{m} / \mathrm{sec})$ & $0.75 \pm 0.25$ & $0.80 \pm 0.30$ & 0.122 \\
\hline A velocity (m/sec) & $0.70 \pm 0.23$ & $0.59 \pm 0.35$ & 0.002 \\
\hline E deceleration time (msec) & $188.8 \pm 72.0$ & $156.4 \pm 70.1$ & 0.000 \\
\hline Body mass index $\left(\mathrm{Kg} / \mathrm{m}^{2}\right)$ & $27.7 \pm 6.4$ & $26.9 \pm 6.2$ & 0.305 \\
\hline LV mass $(\mathrm{Kg})$ & $183.2 \pm 88.6$ & $217.8 \pm 86.0$ & 0.001 \\
\hline Relative wall thickness & $0.41 \pm 0.13$ & $0.34 \pm 0.14$ & 0.000 \\
\hline End-Diastolic volume BSA $\left(\mathrm{cm}^{3} / \mathrm{m}^{2}\right)$ & $69.4 \pm 31.4$ & $99.9 \pm 45.3$ & 0.000 \\
\hline Pulse pressure (mmHg) & $54.3 \pm 18.9$ & $41.6 \pm 15.0$ & 0.000 \\
\hline LV mass height $2.7\left(\mathrm{~g} / \mathrm{m}^{2.7}\right)$ & $48.3 \pm 23$ & $55.4 \pm 18.8$ & 0.009 \\
\hline Body surface area (Monstellar) (m²) & $1.84 \pm 0.18$ & $1.84 \pm 0.24$ & 0.986 \\
\hline Body surface area (Dubois) $\left(\mathrm{m}^{2}\right)$ & $1.81 \pm 0.17$ & $1.81 \pm 0.22$ & 0.875 \\
\hline LV Mass $^{\text {BSA }}\left(\mathrm{kg} / \mathrm{m}^{2}\right)$ & $102.5 \pm 49.4$ & $122.5 \pm 44.7$ & 0.001 \\
\hline
\end{tabular}




\section{Conclusions}

The audit of the ECHO lab showed that the percentage of appropriate indications for ECHO is relatively low and the inappropriate indications unduly high. However, $20 \%$ of people who were referred for hypertension had left atrial enlargement, a feature of hypertensive heart disease. This suggests that hypertension may be re-classified as an appropriate indication for ECHO, at least in Africans. There is a need to corroborate this finding in other studies.

\section{Limitations}

The profile of patients referred for ECHO to the lab was not exhaustive. Important information such as occupational status, level of education, source of funding for $\mathrm{ECHO}$, and even patient satisfaction with the services rendered at the ECHO lab are pivotal pieces of information that make for a holistic assessment of the functioning of the lab. These are not routinely nor periodically collected.

This study was also limited by the available data and the medical record system that is presently operating in the hospital, where individual case notes have to perused to see if each ECHO did have a positive, pivotal impact on patient management or not. This could be easy to do if an electronic medical record system were operational and checklists in this regard were incorporated into the day-to-day functioning of the hospital.

\section{References}

1. Krishnamoorthy VK, Sengupta PP, Gentile F, Khandheria BK. History of echocardiography and its future applications in medicine. Crit Care Med 2007;35:S309-13.

2. Kolo P, Sanya E, Omotoso A, et al. The role of echocardiography in the management of stroke. West Afr J Med 2011;29.

3. Dolan AL, Mishra MB, Chambers JB, Grahame R. Clinical and echocardiographic survey of the Ehlers-Danlos syndrome. Rheumatology 1997; 36:459-62.

4. Brown OR, Demots H, Kloster FE, et al. Aortic root dilatation and mitral valve prolapse in Marfan's syndrome. Circulation 1975;52:651-7.

5. Hossack KF, Leddy CL, Johnson AM, et al. Echocardiographic findings in autosomal dominant polycystic kidney disease. $\mathrm{N}$ Engl J Med 1988;319:907-12.

6. Ploch L. Nigeria: Current issues and U.S. Policy. Congr Res Serv 2013. Available from: https://www.everycrsreport. com/files/20130424_RL33964_3cc7b0c7eaaf1fbf4ea3f78d5d 49a0de88b81b43.pdf

7. Fred HL. Hyposkillia: deficiency of clinical skills. Texas Hear Inst J 2005;32:255-7.

8. American College of Cardiology Foundation Appropriate Use Criteria Task Force, American Society of Echocardiography, American Heart Association, et al. ACCF/ASE/AHA/ASNC/HFSA/HRS/SCAI/SCCM/SCCT/S CMR 2011 Appropriate Use Criteria for Echocardiography. A Report of the American College of Cardiology Foundation Appropriate Use Criteria Task Force, American Society of Echocardiography, American Heart Associat. J Am Soc Echocardiogr Off Publ Am Soc Echocardiogr 2011;24:229-67.

9. Lang RM, Bierig M, Devereux RB, et al. Recommendations for chamber quantification: a report from the American Society of Echocardiography's Guidelines and Standards Committee and the Chamber Quantification Writing Group, developed in conjunction with the European Association of Echocardiography, a branch of the European Society of Cardiology. J Am Soc Echocardiogr 2005;18:1440-63.

10. Oyedeji AT, Akintunde AA, Owojori OO, Peter JO. Spectrum of echocardiography abnormalities among 168 consecutive referrals to an urban private hospital in South-Western Nigeria. Clin Med Insights Cardiol 2014;8:CMC.S14320.

11. Cuspidi C, Rescaldani M, Sala C. Prevalence of echocardiographic left-atrial enlargement in hypertension: A systematic review of recent clinical studies. Am J Hypertens 2013;26:456-64.

12. Patil HR, Coggins TR, Kusnetzky LL, Main ML. Evaluation of appropriate use of transthoracic echocardiography in 1,820 consecutive patients using the 2011 revised appropriate use criteria for echocardiography. Am J Cardiol 2012;109:1814-7.

13. Mansour IN, Razi RR, Bhave NM, Ward RP. Comparison of the updated 2011 appropriate use criteria for echocardiography to the original criteria for transthoracic, transesophageal, and stress echocardiography. J Am Soc Echocardiogr 2012;25:1153-61.

14. Bailey SA, Mosteanu I, Tietjen PA, et al. The use of transthoracic echocardiography and adherence to appropriate use criteria at a regional hospital. J Am Soc Echocardiogr 2012;25:1015-22.

15. Thomas L, Marwick TH, Popescu BA, et al. Left atrial structure and function, and left ventricular diastolic dysfunction: JACC state-of-the-art review. J Am Coll Cardiol 2019;73:1961-77.

16. Gurzun MM, Ionescu A. Appropriateness of use criteria for transthoracic echocardiography: Are they relevant outside the USA? Eur Heart J Cardiovasc Imaging 2014;15:450-5.

17. Rao G, Sajnani N, Kusnetzky LL, Main ML. Appropriate use of transthoracic echocardiography. Am J Cardiol 2010;105: 1640-2.

18. Marwick TH, Gillebert TC, Aurigemma G, et al. Recommendations on the use of echocardiography in adult hypertension: A report from the European Association of Cardiovascular Imaging (EACVI) and the American Society of Echocardiography (ASE). Eur Heart J Cardiovasc Imaging 2015;16:577-605.

19. Fonseca R, Negishi K, Marwick TH. What is the evidence status of Appropriate Use Criteria (AUC)? Insight from a matching exercise with the guidelines for echocardiography. Intern Med J 2015;45:864-9.

20. Messerli FH, Rimoldi SF, Bangalore S. The Transition From Hypertension to Heart Failure: Contemporary Update. JACC Heart Fail 2017;5:543-51.

21. Vidt DG, Prisant LM. Hypertensive heart disease. J Clin Hypertens 2005;7:231-8.

22. Odia OJ, Jesuorobo DE, Akpa MR, et al. Dominance of hypertensive heart disease in a tertiary hospital in Southern Nigeria: An echocardiographic study. Ethn Dis 2012;22:136-9.

23. Ojji D, Stewart S, Ajayi S, et al. A predominance of hypertensive heart failure in the Abuja Heart Study cohort of urban Nigerians: A prospective clinical registry of 1515 de novo cases. Eur J Heart Fail 2013;15:835-42.

24. Dokainish H, Teo K, Zhu J, Roy A, Alhabib KF, Elsayed A, et al. Heart Failure in Africa, Asia, the Middle East and South America: The INTER-CHF study. Int J Cardiol 2016;204:13341. 